\title{
Progress in Laser Risk Reduction for 1 micron lasers at GSFC
}

\author{
William S. Heaps \\ NASA Goddard Space Flight Center \\ Laser and Electro-Optics Branch, 550.0 \\ Greenbelt, MD 20771
}

Abstract-In recent years, lasers have proven themselves to be invaluable to a variety of remote sensing applications. LIDAR techniques have been used to measure atmospheric aerosols and a variety of trace species, profile winds, and develop high resolution topographical maps. Often it would be of great advantage to make these measurements from an orbiting satellite. Unfortunately, the space environment is a challenging one for the high power lasers that would enable many LIDAR missions. Optical mounts must maintain precision alignment during and after launch. Outgassing materials in the vacuum of space lead to contamination of laser optics. Electronic components and optical materials must survive the space environment, including a vacuum atmosphere, thermal cycling, and radiation exposure. Laser designs must be lightweight, compact, and energy efficient. Many LIDAR applications require frequency conversion systems that have never been designed or tested for use in space. For the last six years the National Aeronautical and Space Administration (NASA) has undertaken a program specifically directed at addressing the durability and long term reliability issues that face space-borne lasers. The effort is shared between NASA Goddard Space Flight Center in Greenbelt, Maryland, and NASA Langley Research Center in Hampton, Virginia.
This paper is an overview of the issues facing space-borne lasers and the efforts that Goddard has been pursuing to address them.

\section{INTRODUCTION}

The value of lasers and LIDAR techniques in remote sensing of the Earth and its atmosphere has been proven beyond question over the years. Measurements made from the ground and from aircraft are widely used and are key tools to understanding our planet. LIDAR techniques have been used to measure atmospheric aerosols and a variety of trace species, profile winds, and develop high resolution topographical maps. Clearly, there is much to be gained by making LIDAR measurements from space. An orbiting spacecraft has a wider field of view, can cover hundreds of kilometers per hour, and has access to the full vertical profile of the atmosphere. Successful space based LIDAR missions have generated detailed profilometry of clouds and aerosols over Earth and exquisite topographical maps of Earth, Mars and the Moon. Unfortunately, there have also been serious set backs due to the generally low maturity level of electrooptic materials and components for use in space. This has even resulted in on-orbit failures and program cancellation. The most problematic element of a space based LIDAR system is the laser itself. 
In 2002 the National Aeronautics and Space Administration (NASA) implemented the Laser Risk Reduction Program (LRRP) to specifically address the reliability issues that affect lasers in space. The work is being conducted jointly by NASA Goddard Space Flight Center (GSFC) in Greenbelt, Maryland and at NASA Langley Research Center (LaRC) in Hampton, Virginia. The purpose of this paper is to give an overview of what risk factors affect space based lasers and LIDAR systems and what activities have been undertaken within this program to address those risks. The Goddard portion of this program is scheduled to conclude in the fall of 2007 and the Langley effort a year later.

\section{THE SPACE ENVIRONMENT}

It is not difficult to find turn-key laser systems suited to many ground based LIDAR applications which require little maintenance over the life of the system. Space based LIDAR systems are entirely a different matter. Although the International Space Station and the Hubble Space Telescope can be serviced while on orbit, it is generally out of the question to service an instrument on an Earth orbiting spacecraft, never mind one bound for Mars. Long term reliability is crucial.

The first challenge for an instrument to survive is the launch itself. Although NASA has been launching delicate optical instruments for decades, the tuned resonant cavity of a laser can be far more sensitive to the demands of launch than most telescopes. The Delta II, a widely used launch vehicle, can subject its payload to accelerations in excess of $10 \mathrm{~g}$ 's and acoustic levels in excess of $140 \mathrm{~dB}$, which is well above the pain threshold for humans [1].
Many of the desired LIDAR applications to be implemented in space will require high average output powers. This may be required due to long optical path length, competition with solar light, or low concentration of the chemical species being investigated. A laser head with high average output power will result in a considerable heat load on the spacecraft. Ground based laboratory systems frequently include a chilled water loop to remove the heat from the laser head. Pumping fluids in zero gravity and over a wide variety of temperature regimes has been found to be impractical. Developing techniques for transferring heat from a compact laser head to other regions of the spacecraft for radiation to space is another challenge faced by designers of these systems. The vacuum of space increases the difficulty of this task due to the loss of the thermal conduction path normally provided by air

The vacuum environment of space also dramatically increases the risk of contamination and optical damage in a laser, especially for missions with long cruises or operational lifetimes. Adhesives, lubricants, electrical insulation, and any number of other materials used in the construction of the spacecraft will outgas in the vacuum of space. The outgassed material can deposit on the laser optics and may absorb energy from the laser beam and subsequently resuit in burning of the laser optics. Once the laser optics are damaged, the performance of the laser can be seriously reduced or even destroyed. Simply using the wrong adhesive in an instrument can result in complete loss of a mission.

The radiation exposure suffered by spaceborne instruments is another serious issue. Most optical materials forn color centers on exposure to radiation which results in darkening of the optic. Although radiation darkening has been measured for 
many materials, there is not much data on many of the newer electro-optic materials, particularly those used for frequency conversion.

The overall reliability of manufactured electro-optic components in the space environment has not been weil established. Most of these products are not designed by their manufacturer with the space environment in mind. Conducting the necessary tests to qualify manufactured products for use in space is a laborious process. It is further complicated by the fact that vendors continuously improve and change their products, making earlier test data less valuable. Developing appropriate tests and screening techniques for ensuring long term reliability of manufactured products in the space environment is very important. For example, it has been determined by NASA that the premature failure of laser diode arrays caused the loss of the first of three lasers on board the Geoscience Laser Altimeter System (GLAS) instrument on board the Ice, Cloud and land Elevation Satellite (ICESAT) now orbiting Earth [2].

Many of the desired LIDAR applications require laser wavelengths other than 1.0 micron. Thus far, the 1.0 micron diode pumped solid state Nd:YAG laser has been the workhorse laser for space. Clearly the technology for generating other wavelengths is available in ground based systems, but few have been developed for use in space. Developing a space qualified 2.0 micron laser will enable coherent detection of winds and water currents. Developing space qualified frequency conversion systems will enable $\mathrm{CO} 2$, ozone, and other spectroscopic LIDAR missions.

On top of all these issues are the added design constraints of volume, mass and power consumption. All space based instruments must be compact, lightweight and operate on a minimum of electrical power.

\section{OUTLINE OF THE PROGRAM}

NASA implemented the LRRP to investigate the root causes of failures in laser systems designed for space and develop robust laser architectures that can be adapted to the specific requirements of future missions. This was done in response to an external Earth Science Independent Laser Review Panel which recommended that NASA intensively develop critical laser/LIDAR technology elements before mission approval, since development during fast-track missions presented unacceptable levels of risk [3]. The LRRP is a joint activity that has been co-funded by the NASA Earth Science Technology Office (ESTO) and NASA Enabling Concepts and Technologies (ECT), Advanced Measurement \& Detection Program.

The LRRP has been conducted as a partnership between NASA GSFC and NASA LaRC, with each center addressing the issues most suited to their strengths. A number of industrial and academic partners are also participating in the program. The LRRP can be thought of as being comprised of three basic areas of research: development of space qualified lasers, investigation of reliability issues and failure modes, and the development of ancillary hardware technologies for spaceborne LIDAR systems. In addition to the technical research, we are conducting a knowledge management effort to facilitate dissemination of the valuable information developed on this program. 


\section{A. Laser Development}

When the LRRP was first being proposed, a survey of desired spacebased LIDAR missions was conducted. It was determined that there were six categories of measurements of high priority to earth science: coherent wind detection, noncoherent wind detection, coherent detection of river/ocean surface currents, altimetry/bathymetry, and range resolved measurements of ozone and $\mathrm{CO} 2$ using the differential absorption technique. It was decided that all six of these measurements could be enabled by the development of two primary laser systems operating at 1.0 micron and 2.0 micron. These would then be combined with a limited number of frequency conversion methods [4]. GSFC has pursued the 1.0 micron system while LaRC has concentrated on the 2.0 micron laser.

The fundamental goal is to develop knowledge about building space qualified lasers. To achieve this, we are designing, and building testbed lasers which will then be subjected to rigorous environmental testing required for space qualification. Although future LIDAR missions may not use the exact architectures produced under this program, we will have developed the baseline knowledge of how to design and build successful spaceborne laser systems. For both laser systems under development it was decided to target 1.0 Joule/pulse output energy. Some of the desired measurements require substantially less energy per pulse, but may require high repetition rates. Rather than spread resources too thin it was decided to focus on the high energy per pulse system, which was regarded as the more challenging problem to solve.

The laser being developed at $\mathrm{LaRC}$ is a diode pumped $\mathrm{Ho}$, Tm:LuLF rod laser producing 2.0 micron light. This laser has demonstrated $>1.05 \mathrm{~J}$ Q-switched output energy in a partially conductively cooled head. It employs one power oscillator and two amplifiers using a double pulse format. It is the first Q-switched 2-micron laser exceeding $1 \mathrm{~J}$ output energy and is one order of magnitude higher in energy than any laser previously demonstrated by other groups. This design was more recently adapted to produce the first fully conductively cooled 2-micron pulse laser.

The 1.0 micron laser being developed at GSFC is a diode pumped Nd:YAG slab laser. GSFC has a considerable track record in developing 1.0 micron lasers for space, having developed the $100 \mathrm{~mJ} /$ pulse lasers for the GLAS/ICESat mission and the 20 $\mathrm{mJ} /$ pulse laser for the Mercury Laser Altimeter that was launched as part of the MESSENGER mission to Mercury in August 2004. To achieve $1.0 \mathrm{~J} /$ pulse the GSFC team is developing a system composed of an oscillator followed by three amplifier stages. The oscillator optical design is complete and is currently undergoing tests. The oscillator is stabilized by a reference seed laser developed in partnership with AdvR Corporation. The amplifier stages for the testbed laser are currently under development.

Laser development is not limited to the primary testbed lasers at both GSFC and LaRC. Both centers are developing smaller breadboard lasers and developing computer models of alternative laser designs. Clearly not all LIDAR applications will be able to make use of the 1 Joule/pulse laser architecture, so it is important to keep other concepts under development. However, only the main testbed lasers are being developed into fully packaged units and subjected to environmental tests. 


\section{B. Reliability Issues}

A number of issues are already known to be major factors in the reliability of space based lasers. Many of these issues center around reliability of materials or manufactured products. Others are related to processes and handling during the build, test, and integration of instruments.

One of the most important reliability efforts underway is the evaluation of high power laser diode arrays used as the laser pump source. Since the 1.0 and 2.0 micron lasers each require different pump wavelengths and are designed to operate under substantially different conditions, both GSFC and LaRC have set up diode test and evaluation facilities.

Diodes from various vendors have been put through an exhaustive series of inspections and checkout procedures, some of which are high magnification photos, thermal imaging of the diode arrays while operating, optical output power versus current measurements,
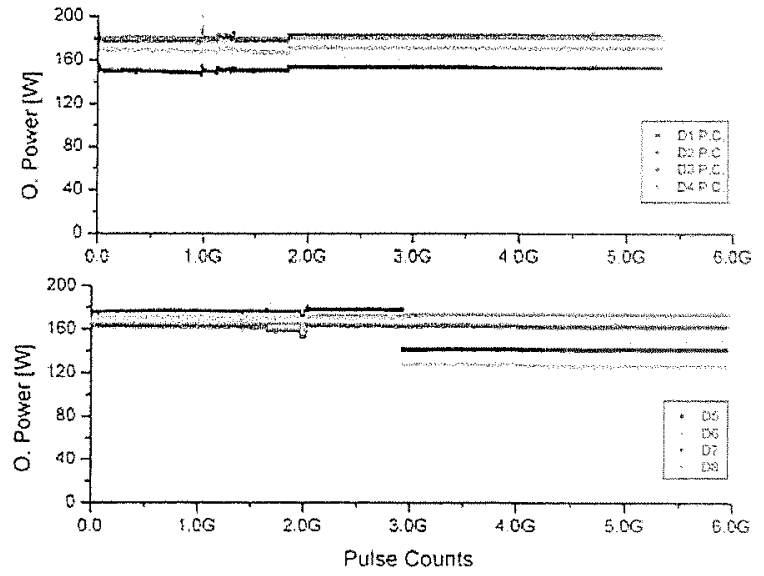

Figure 1. This illustrates results for a life test on some $\mathrm{G} 4$ diode arrays. Diodes at top are power cycled off 2 minutes out of 20 . Diodes at bottom have run contiuously. Decreases in lower figure represent bar failures.

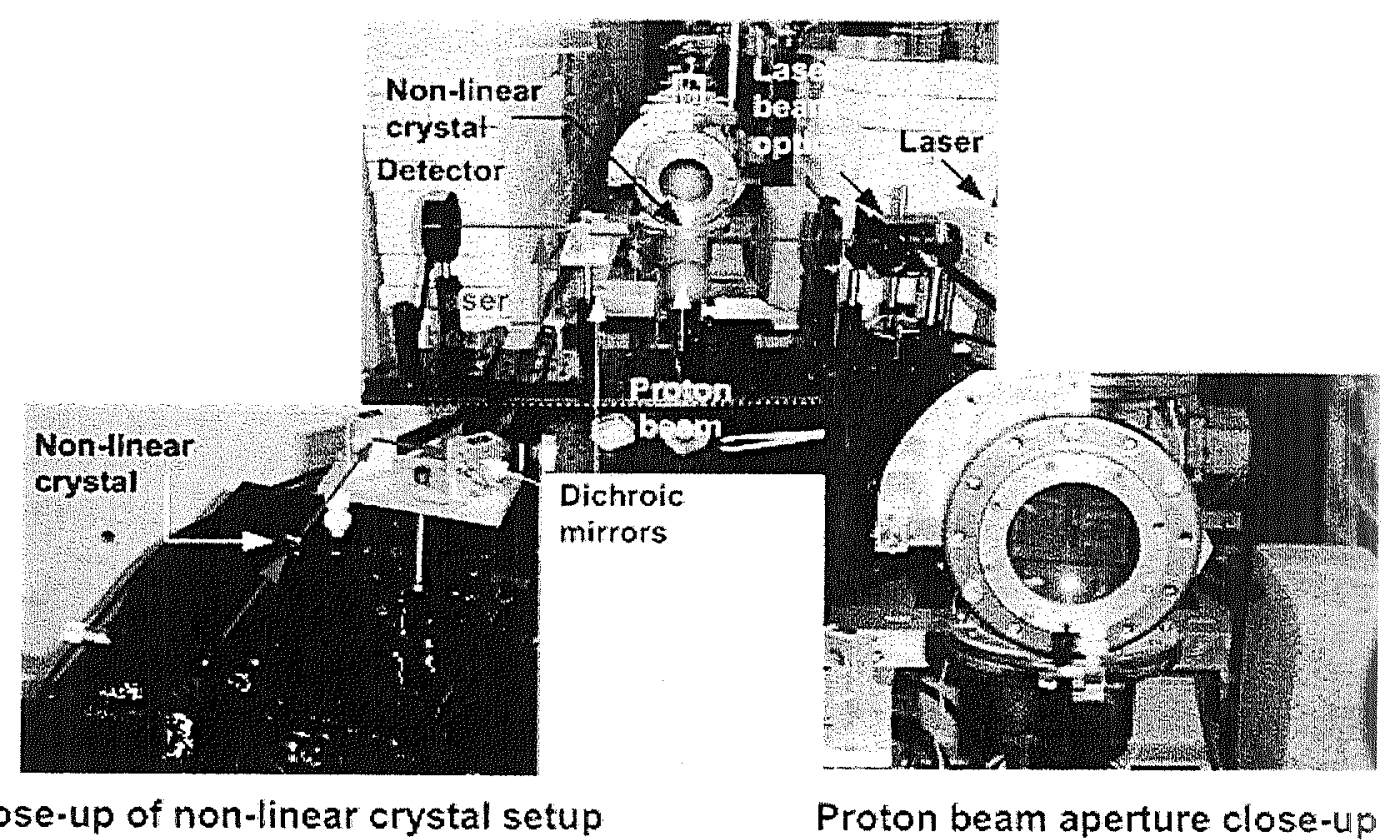

Figure 2. Non-linear material rad test. 
and spectral profiling. Once the diodes have been fully characterized they are placed in a long term life test whichsubjects the diodes to test. operational conditions similar to existing space flight programs. At designated life intervals the diodes are removed from the life test, re-characterized, and returned to the characterization tests As failures develop, they are reviewed to determine whether early data could have predicted the failure. The goal is to develop a battery of screening tests that will enable NASA to hand pick laser diode arrays from those provided by a vendor. We also work closely with the vendors to address failure modes and refine manufacturing processes. Both the laser diodes and the nonlinear optical materials have been subjected to radiation tests that included beta (cobalt $60)$ proton exposure. Diodes have proven to be robust. Most non-linear materials suffer darkening which may be reversible. Studies at the Montana State University are underway to examine the damage in greater detail

Contamination is one of the leading concerns related to space flight lasers. This is because the resulting damage is so catastrophic and widespread. Many contamination related failures have been suffered during ground testing of flight lasers. This is obviously a costly way to determine what materials are to be considered contaminants. Under the LRRP we have conducted a number of tests ini which a high energy laser is fired at a test window inside a vacuum chamber. A compound under test is released into the test chamber and allowed to deposit on the test window.

The window is monitored for optical damage as a function of contaminant level, laser energy fluence, and number of laser shots. The goal was to develop a database of laser induced damage thresholds for as many relevant compounds as possible. This database will be used as a resource in developing approved materials lists for future flight laser programs. It will also be used to determine minimum cleanliness levels.

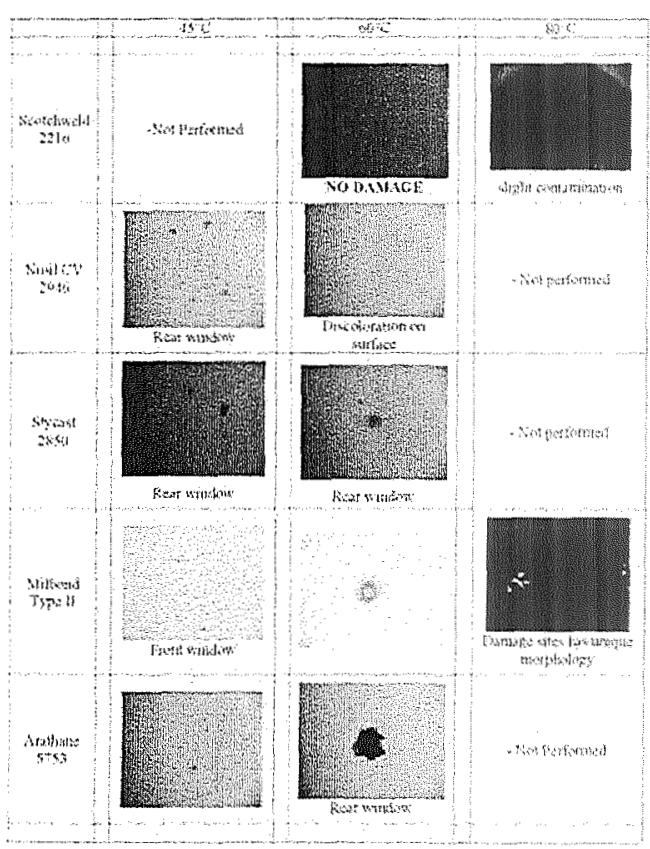

Figure 3. This illustrates the nature of damaged produced by the presence of some commonly used adhesives and solvent

\section{Space LIDAR enabling hardware}

Although the laser itself is considered the primary risk associated with space based LIDAR technology, there are other components of a LIDAR system that clearly need to be made ready for space. Among these are improved detectors, receiver systems and frequency conversion systems.

For most LIDAR applications, an improvement in the optical efficiency of the 
detector results in a corresponding reduction in the output power required by the laser itself. This has added system level benefits by reducing electrical power, thermal load, size, and weight. Both LaRC and GSFC are working with industrial and academic partners to develop avalanche photodiode detectors that have high sensitivity and low noise levels. LaRC is working with partners to develop In $\mathrm{GaSb}$ detectors for use in the $2.0-2.5$ micron range while GSFC is working with partners to improve the technology of InGaAs detectors for use in the 1.0-2.0 micron range [5].

Ozone profiling will require the development of space qualified frequency conversion technology that can convert 1.0 micron light to $308 \mathrm{~nm}$ and $320 \mathrm{~nm}$. The high average power that will be required to penetrate the atmosphere presents a. challenging problem for such a system

Two approaches are being pursued. LaRC has partnered with Sandia National

Laboratory to pursue an optical parametric oscillator (OPO) system capable of $200 \mathrm{~mJ}$ pulses at $10 \mathrm{~Hz}$. GSFC has partnered with ITT Advanced Engineering and Science Division (ITT AES) to develop an OPO system capable of $10 \mathrm{~mJ}$ pulses at $1.0 \mathrm{kHz}$ [5].

GSFC has also been working with ITT AES to develop an optical parametric amplifier system to convert 1.0 micron light to 1.55 microns for use in $\mathrm{CO} 2$ profiling. The system is designed to produce an average power of $\sim 2.0 \mathrm{~W}$ at a pulse repetition rate of $10 \mathrm{kHz}$ and have a spectral line width of $70 \mathrm{MHz}$ HWHM [5]. This system is scheduled to be delivered in early April.

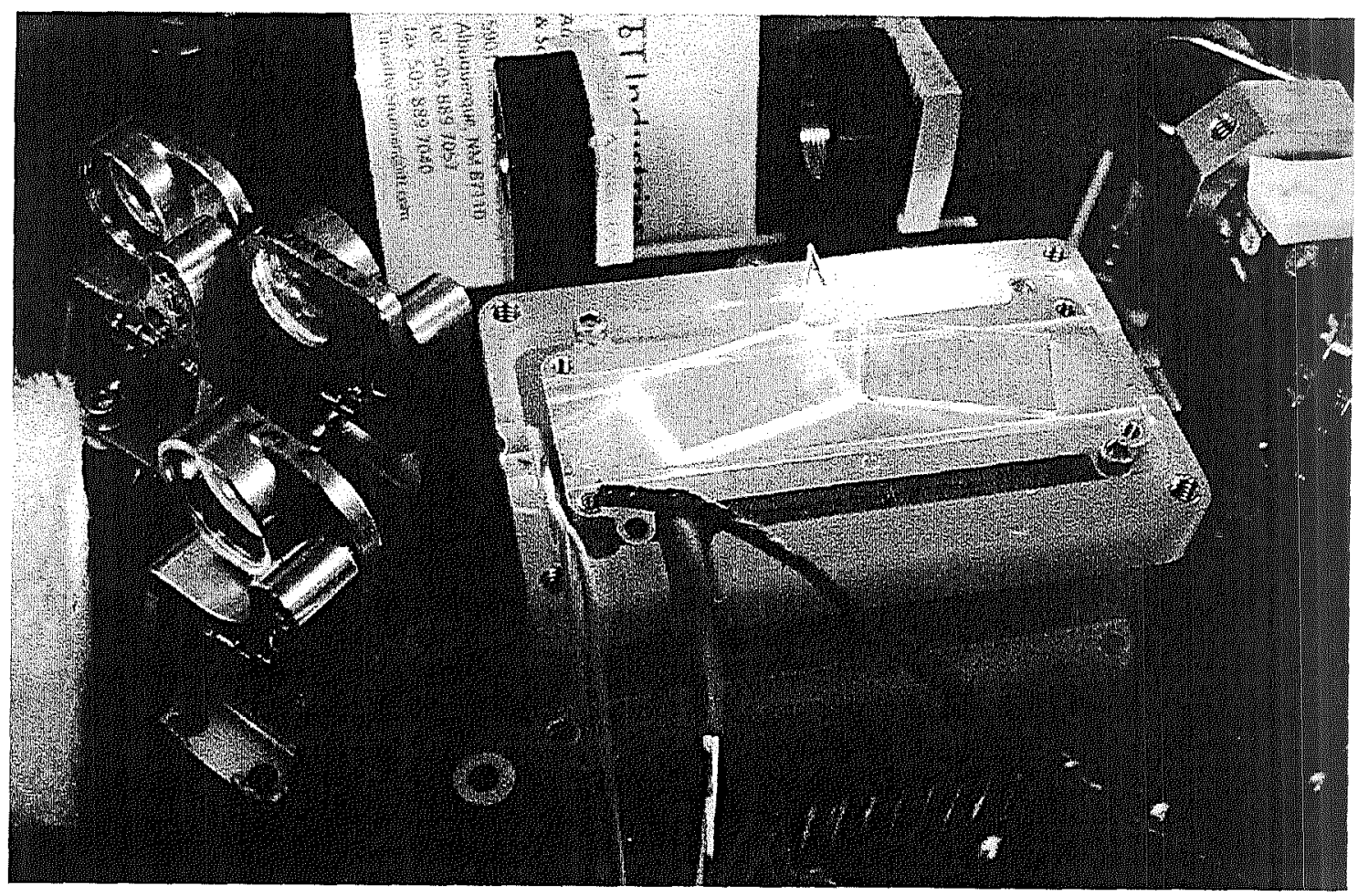

Figure 4. The 1.55 micron OPA developed by ITT. 


\section{Knowledge management}

It is important to understand that the primary deliverable of the LRRP is knowledge. Even in the hardware oriented tasks the goal is to better understand how to design and build space qualified systems. Thus, it is critical to disseminate the knowledge developed under the LRRP within NASA, to other government agencies with spaceborne laser programs, and to our industrial and academic partners. The LRRP is developing a comprehensive electronic database of spaceborne laser related knowledge. A web based interface has been designed at GSFC so that users can easily search for relevant information.

\section{CONCLUSION}

LIDAR has proven to be a critical tool for remote sensing of Earth and other planets. It is clear that making LIDAR measurements from space will eventually become a routine matter. Previous missions have proven that the laser is the highest risk element in a spaceborne LIDAR system. Through the LRRP, NASA seeks to meet the needs of the science community by developing the knowledge and technologies that will ensure that future space based LIDAR missions will be successful.

\section{REFERENCES}

[1] Delta II Payload Planners Guide, MDC H3224C, McDonnel Douglas Aerospace, October 1993.

[2] Community Forum on Laser Diode Arrays in Space-Based Applications, Arlington, VA, March 2-3, 2004.

[3] Earth Science Independent Laser Review Panel Report, S. B. Alejandro, R. M. Hardesty, J. Hicks, D. Killinger, and M. Lapp, 27 Nov. 2000.

[4] Laser Risk Reduction Program FY04 Task Agreement, William S. Heaps, Upendra N. Singh, October, 2006 .

[5] Laser Risk Reduction Mid-Phase Review and Technical Interchange Meeting, Greenbelt, MD, February 11 12, 2004. 